\title{
Should we ignore what we cannot measure? How non-uniform stretch, non-uniform wall thickness and minor side branches affect computational aortic biomechanics in mice.
}

\author{
Mauro Ferraro, $\mathrm{PhD}^{1}$, Bram Trachet, $\mathrm{PhD}^{1,2}$, Lydia Aslanidou1, \\ Heleen Fehervary ${ }^{3}$, Patrick Segers, PhD ${ }^{2}$, Nikolaos Stergiopulos, PhD ${ }^{1}$
}

${ }^{1}$ Institute of Bioengineering, Ecole Polytechnique Fédérale de Lausanne, Lausanne, Switzerland ${ }^{2}$ IBiTech - bioMMeda, Ghent University, Ghent, Belgium

${ }^{3}$ Biomechanics Section, University of Leuven, Leuven, Belgium

Mauro Ferraro

LHTC STI IBI EPFL

MED 32924 Station 9

$\mathrm{CH}-1015$ Lausanne (Switzerland)

Tel: +41 216938342

mauro.ferraro@epfl.ch 
This is a pre-copyedited, author-produced PDF of an article accepted for publication in Annals of Biomedical Engineering following peer review.

The version of record is available online at:

Should we ignore what we cannot measure? How non-uniform stretch, non-uniform wall thickness and minor side branches affect computational aortic biomechanics in mice.

Mauro Ferraro, Bram Trachet, Lydia Aslanidou, Heleen Fehervary, Patrick Segers, Nikolaos Stergiopulos.

Annals of Biomedical Engineering 2018, 46(1), 159-170

doi: 10.1007/s10439-017-1945-y 


\begin{abstract}
Aim: To advance the state-of-the-art in computational aortic biomechanics, we investigated the influence of (i) a non-uniform wall thickness, (ii) minor aortic side branches and (iii) a non-uniform axial stretch distribution on the location of predicted hotspots of principal strain in a mouse model for dissecting aneurysms.

Methods: After 3 days of Angiotensin II infusion a murine abdominal aorta was scanned in-vivo with contrast-enhanced micro-CT. The animal was subsequently sacrificed and its aorta was scanned ex -vivo with Phase-contrast X-Ray Tomographic Microscopy (PCXTM). An automatic morphing framework was developed to map the non-pressurized, non-stretched PCXTM geometry onto the pressurized, stretched micro-CT geometry. The output of the morphing model was a structural FEM simulation where the output strain distribution represents an estimation of the wall deformation, not only due to the pressurization, but also due to the local axial stretch field. The morphing model also included minor branches and a mouse-specific wall thickness. A sensitivity study was then performed to assess the influence of each of these novel features on the outcome of the simulations. The results were supported by comparing the computed hotspots of principal strain to hotspots of early vascular damage as detected on PCXTM.

Results and Conclusions: Non-uniform axial stretch, non-uniform wall thickness and minor subcostal arteries significantly alter the locations of calculated hotspots of maximal principal strain. Even if experimental data on these features are often not available in clinical practice, one should be aware of the important implications that simplifications in the model might have on the final simulated result.
\end{abstract}




\section{Introduction}

In the last three decades, the employment of computational biomechanics for the study of pathologies leading to aortic dilatation, e.g. abdominal aortic aneurysms (AAA) or aortic dissection, has become increasingly widespread (1). Recent advances in medical imaging, mathematical modeling, and computational capacity have enabled our community to come up with significant contributions in the understanding of progression and rupture of a degenerating aortic wall. Studies approaching this problem focus either on the disturbed hemodynamics (2), on the stress distribution of the vascular wall under pathophysiological conditions (3), or, more recently, on the growth and remodeling phenomena that occur during aneurysm development (4). Of these different computational approaches, finite element rupture prediction models are the most clinically relevant as the computed stress-strength ratio can be used as a tool to identify small aneurysms at risk of rupture (5).

AAAs and dissections are, however, often asymptomatic. Therefore data are usually only collected in humans when the lesion has already reached an advanced stage. A recent modeling trend to overcome the lack of data on AAA initiation is to use animal models as a complementary tool to classical human studies. Due to the availability of genetically modified strains, the rapid time course of disease development and the fact that longitudinal data can be obtained both in-vivo and ex-vivo, these models can offer unique insights into disease initiation and progression (6; 7). The angiotensin Il-infused mouse model, in which AAA formation is promoted by infusion of the vasoconstrictive peptide hormone angiotensin II, has become the model of choice for preclinical research on AAA (8). Despite the fact that this model presents many similarities with human AAA such as elastin degradation and luminal dilatation (9; 10), dissecting AAAs in Ang-II infused mice develop suprarenally rather than infrarenally and dilate due to a dissection rather than a circumferential wall degradation (11). A recent study from our group used a novel synchrotron-based imaging technique (Phase Contrast X-ray Tomographic Microscopy; PCXTM) to investigate dissecting AAA initiation in these angiotensin II-infused mice (12). PCXTM employs X-ray synchrotron radiation to obtain images with pixel sizes in the micrometer-scale that have sufficient soft tissue contrast to differentiate between most constituents of the aortic wall. Our PCXTM-based images demonstrated that dissecting AAAs start with micro ruptures in the media, which could be visualized since the contrast agent that had been injected during in-vivo micro-CT locally infiltrated the aortic wall (13). These micro-ruptures occurred predominantly near suprarenal side branches such as the celiac and mesenteric arteries. The concentration of micro-ruptures near specific side branches suggested that the local mechanical equilibrium along the abdominal aorta may play an important role to determine the regions where dissecting AAAs initiate.

From a computational viewpoint, we investigated the hypothesis that biomechanics play a role in the initiation of dissecting AAAs through the evaluation of the mechanical forces acting on the murine vascular wall developing a fluid-structure interaction (FSI) simulation on a mouse-specific geometry (14). But while these simulations showed promising outcomes the FSI framework incorporated several limitations. A uniform wall thickness was used, minor side branches were neglected and axial pre-stretch was not included. Each of the limitations mentioned above affect the current state-of-the-art in biomechanical modeling of the aorta, not only in a preclinical context, 
but also for simulations that are obtained in a clinical context, such as AAA rupture predictions. Not all of these limitations receive, however, the same amount of attention. The lack of minor side branches and axial pre-stretch are rarely discussed in literature and often ignored altogether, while a lot of attention is given to the importance of the material model. Therefore the goal of this manuscript is twofold: we aim to (i) take advantage of the superior imaging that has recently become available in a pre-clinical setting to advance the state-of-the-art in aortic biomechanics and (ii) investigate to what extent the resulting technical improvements influence the final result.

To this end we present a novel computational framework that was developed in order to map the non-pressurized, non-stretched ex-vivo PCXTM scans (which incorporate mouse-specific thickness and minor side branches) onto the pressurized, stretched in-vivo micro-CT scans (which only represent the aortic lumen). Due to the detailed information in the pre-clinical synchrotron images, the resulting morphing simulation allowed us to embed the mouse-specific wall thickness, minor subcostal branches, and local axial stretch distribution into a single structural model. Moreover this animal showed micro-ruptures after 3 days of Ang II-infusion, which allowed for a one-on-one comparison of predicted regions of high strain to experimentally observed wall damage. Our approach thus addresses all of the limitations mentioned above, except for the material model. We subsequently performed a sensitivity analysis in which we assessed to what extent the inclusion of each of these novel features influenced the location and magnitude of predicted hotspots.

\section{Material and Methods}

In this Section, the morphing computational framework is described, including the experimental protocol, preprocessing, meshing procedure, and simulation set-up.

\section{Experimental Protocol}

All the procedures were approved by the Ethical Committee of Canton Vaud, Switzerland (EC 2647.2) and performed according to the guidelines from Directive 2010/63/EU of the European Parliament on the protection of animals used for scientific purposes. , a male ApoE-deficient mouse on a C57BI/ 6 background was implanted with a $200 \mu$ osmotic pump, filled with a solution of angiotensin II in saline $0.9 \%$ as previously described (6). Prior to implantation the pump was primed for 24 hours at 37 degrees. The mouse was sacrificed after 3 days of Ang II infusion to investigate the early phase of dissecting aneurysm development. Care was taken not to damage the aortic tissue during the dissection of the aorta while surrounding connective tissue was carefully removed. The following imaging techniques were used in this study:

(i) In-vivo micro-CT imaging. Contrast enhanced micro-CT was taken at day 3. The animal was injected intravenously in the lateral tail vein with $4 \mathrm{~mL} / \mathrm{g}$ body weight of ExiTron nano 12000 (Miltenyi Biotec, Bergisch Gladbach, Germany) and subsequently scanned in vivo with a Quantum FX micro-CT scanner (Caliper Life Sciences, Hopkinton, MA, USA). During micro-CT imaging the animal was anaesthetized by inhalation of $1.5 \%$ isoflurane. $(12 ; 13)$. This technique provides an isotropic pixel size of $50 \mu \mathrm{m}$ of the abdominal aorta lumen, but the tissue contrast is not sufficient to provide information about the wall 
thickness. The scan was not gated and the generated micro-CT segmentation represents an average between systolic and diastolic geometries;

(ii) Ex-vivo PCXTM imaging. After sacrifice, the abdominal aorta was excised and the sample was fixed by immersion in freshly prepared $4 \%$ paraformaldehyde (PFA) at $4^{\circ} \mathrm{C}$ temperature for 24 hours. The samples were scanned at the TOMCAT beamline of the Swiss Light Source, Paul Scherrer Institut, Villigen, Switzerland as previously described (12). PCXTM computer micro-tomography provides volumetric data of samples in a nondestructive way with an isotropic pixel size of $6.5 \mu \mathrm{m}$. This novel imaging technique yields enough tissue contrast to accurately evaluate the wall thickness and to segment small subcostal side branches (15).

\section{Preprocessing}

Both in-vivo micro-CT and ex-vivo PCXTM scans were semi-automatically segmented in the software package Mimics v.17.0 (Materialise, Leuven, Belgium). For micro-CT scans this required manual intervention to separate aortic and venous segments of the contrast-enhanced vasculature. The cross-sectional diameter of the smallest side branches (e.g. subcostal arteries) only consisted of 35 pixels in the micro-CT images. In case of doubt, the PCXTM image stack was used as a guidance to guarantee that the correct structures were identified. For PCXTM the contrast agent infiltrations within the vessel wall were segmented separately (Fig. 1 a) since previous observations had showed that such infiltrations represent a clear indication of early vascular damage (13). Both micro-CT and PCXTM-based masks were smoothed while taking care not to cause any shrinkage. The open-source library VMTK (16) was subsequently used to finalize pre-processing. Inner and outer walls of PCXTM were separated in order to generate the ex-vivo computational mesh, while the side branches of the PCXTM model were removed and reconstructed. Since we lack any reference on the side branches, we decided to develop a consistent extension technique. Each side branch was artificially extended by means of a branch-specific extension length proportional to the corresponding one measured in the CT model. In particular, the distance between the celiac and mesenteric bifurcation was calculated in terms of abscissa coordinate of the centerline, both in the CT and PCXTM scans. The ratio between these two quantities provided an extension coefficient which was multiplied by the PCXTM side branch length and used as extension length.

\section{Meshing procedure}

In order to generate the ex-vivo mouse-specific mesh, the outer wall of the PCXTM model was discretized with an unstructured quadrilateral mesh (17). The outer mesh was then smoothed using a non-shrinking Taubin smoothin filter (passband 0.1, 100 smoothing iterations) and projected onto the mesh of the inner wall through an in-house developed Matlab code (The Mathworks Inc., Natick, MA, USA). The result was an unstructured hexahedral solid mesh of the ex-vivo geometry (Fig. 1d). All meshes were auto-generated to ensure grid independency of the simulation results, and the details of the final, converged mesh can be found in Table 1. The average element edge length in the converged models is $9.6 \mu \mathrm{m}$, while the maximum and minimum lengths are $34 \mu \mathrm{m}$ and $1.5 \mu \mathrm{m}$, 
respectively.

\section{Morphing framework set-up}

The evaluation of the local stretch field along the abdominal aorta requires the generation of a pointwise correspondence between the ex-vivo PCXTM mesh (not stretched, not pressurized) with the in-vivo CT model (in-vivo axial stretch, pressurized). To this end we implemented a branch-based change of coordinates. Using VMTK, branch splitting was performed on the micro-CT model and the inner wall of the PCXTM mesh (Fig. 2a). At this stage, each branch of the arterial network was topologically equivalent to a cylinder and a rectangular parametric space could be generated. In the circumferential direction, the angular position of each point on the surface mesh was parameterized with respect to the centerline following the method proposed in (18) (Fig. 2b). In longitudinal direction, a parameterization was performed using the harmonic mapping method presented in (19). In particular, a Laplacian partial differential equation was solved on the surface by imposing Dirichlet boundary conditions on the two open boundaries, generating a longitudinal parametric distribution (see Fig. 2c).

The combination of the two parametrizations can be compared between micro-CT and PCXTM models, and a standard nearest neighbor approach has been used to find the minimum distance for each PCXTM mesh node with respect to all the micro-CT nodes (20). Once all the maps were computed, the global displacement map was generated using the element connectivity of the PCXTM model as a reference. In order to avoid mesh distortion, smoothing and projection procedures were performed in a similar fashion to those used during the meshing phase.

The final map shows very good agreement with the original micro-CT model (Fig. 2d), with maximum error of $10 \mu \mathrm{m}$ located at the main bifurcations, which is 5 time less than the micro-CT pixel size.

The global map, representing a surface mesh with the shape of the CT model and the mesh size and distribution of the PCXTM inner wall mesh, was employed as a set of displacement conditions to morph the latter onto the former. Both the final PCXTM model and the global displacement map were automatically embedded within an input file for the commercial FEM solver Abaqus/Standard 6.13 (Simulia, Dassault Systemes, Providence, RI, USA). The structural simulation was considered static and fully displacement controlled, under a large deformation regime (Fig. 2e). A nearly incompressible Arruda-Boyce constitutive model was used (Eq. 1). The material parameters $(\mu=24358 \mathrm{~Pa}, \lambda \mathrm{m}=1.1)$ were the same used in the previous FSI work (14).

$$
\begin{gathered}
W=\mu \sum_{i=1}^{5} \frac{\alpha_{i}}{\lambda_{m}^{2 i-2}}\left(I_{1}^{i}-3^{i}\right) \\
\alpha_{1}=\frac{1}{2}, \alpha_{2}=\frac{1}{20}, \alpha_{3}=\frac{11}{1050}, \alpha_{4}=\frac{19}{7000}, \alpha_{5}=\frac{519}{67375 \lambda_{m}^{8}}
\end{gathered}
$$

Where $\mathrm{W}$ is the strain energy per unit reference volume and $\mathrm{I}_{1}$ is the first deviatoric strain invariant. At the open boundaries, all nodes throughout the wall thickness were constrained to follow the motion of the corresponding nodes of the inner wall, in order to avoid unphysical shearing effects. In order to facilitate the comparison of the final outcome with the location of micro-ruptures in the PCXTM model, the strain contour plot was mapped backward onto the ex-vivo undeformed configuration and the artificial extensions in side branches were removed for visualization. 


\section{Sensitivity analysis}

In order to assess which aspects of the morphing approach contribute most to the results, we disentangled the effect of the different features. First, the mouse-specific thickness was replaced with a constant thickness in a morphing approach which still included the small side branches. Second, the minor subcostal arteries were digitally removed in a morphing approach which still included mouse-specific thickness (Fig. $3 \mathrm{~d}$ ). In literature, an approximate $10 \%$ value with respect to the in-vivo diameter is typically used for wall thickness when no ex-vivo data are available $(14 ; 21)$. However, the application of this coefficient on our ex-vivo geometry resulted in a strong underestimation of the mouse-specific wall thickness values. We therefore decided to apply the $5^{\text {th }}$ and the $95^{\text {th }}$ percentile of the local ratio between the real thickness value and the computed diameter along the whole artery. All thickness values are expressed with respect to the local inner diameter of the ex-vivo scan.

\section{Results}

A significantly non-uniform thickness was observed along the aorta in the mouse-specific model (Fig. 3a). Moreover, the transition between the main vessel and the side branches was smooth and continuous. The mouse-specific thickness varied to an average thickness of $0.093 \mathrm{~mm}$ in the nondeformed to $0.046 \mathrm{~mm}$ in the deformed state. This non-uniformity of the morphed model was in sharp contrast with both constant thickness models, which showed a uniform thickness distribution within each branch but a discontinuous transition from the main vessel to the side branches (Fig. $3 \mathrm{bc}$ ). The $5^{\text {th }}$ percentile thickness led to an average thickness of $0.102 \mathrm{~mm}$ in the non-deformed and $0.0217 \mathrm{~mm}$ in the deformed state, corresponding to the $30 \%$ and $6 \%$ with respect to the local diameter, respectively (Fig. 3b). The $95^{\text {th }}$ percentile thickness generated an average thickness of 0.13 $\mathrm{mm}$ in the non-deformed and 0.0529 in the deformed state, corresponding to the $47 \%$ and $10 \%$ with respect to the local diameter, respectively (Fig. 3c).

After the morphing simulation, the distribution of the maximum principal strain was quantified at the outer wall of all the considered models, with particular focus on the region of high strain (termed hotspots hereafter). For the sake of completeness, an additional figure showing the maximum principal strain distribution at the inner wall has been included in the Supplementary Material. Looking at the full model, the values ranged from 0.3 in the most tethered regions close to the mesenteric artery to 1.66 at the orifice of the celiac artery. In addition to the main strain concentration on the ventral side of the celiac bifurcation, two hotspots were detected on the dorsal side of one of the subcostal arteries and on the left-ventral side of the mesenteric artery (Fig. 4a). Two slices of the morphed model corresponding to the contrast agent infiltration regions were selected for a detailed comparison to the local strain concentrations (Fig. 4c). Both the overall 3D assessment and the 2D slices show good agreement between the computed strain and the experimentally observed infiltrations of contrast agent (Figure 4c).

\section{Sensitivity analysis}

Each of the four models that were included in the sensitivity analysis yielded significantly different 
numerical results and none of the simplified models was able to get a strain distribution similar to the one exported from the full model. (Fig 5 and 6 ). While both constant thickness models had a good qualitative agreement with the full model, they were not able to detect the hotspot in the celiac bifurcation (Fig. 5bc). This was even more pronounced when focusing on the zoomed 2D panels, where the intercostal and mesenteric levels showed a good correspondence with the full model while the celiac level did not (Fig. 6bc).

The model without minor side branches had a significantly different strain distribution compared to the full model; especially on the dorsal side, where the support of the subcostal arteries no longer contributed to the final result (Fig. 5a). This was also clear from the 2D slices; the model without minor side branches completely missed the strain concentration in the dorsal side of the subcostal artery, while the strain concentrations at the celiac and mesenteric levels were significantly mitigated (Fig. 6a).

In order to carry out a quantitative comparison between different models we selected a specific area of interest near the celiac bifurcation, which was where the main contrast agent infiltration occurred (Fig 7a). Within this region of interest we exported the max principal strain values and classified them into bins of 0.2 in width (Fig. 7bcd) (22). In the full model the highest number of nodes occurred around a maximal principal strain of 1.3, while this peak occurred at a much lower value for all of the simplified models $\left(5^{\text {th }}\right.$ percentile thickness: $0.9,5^{\text {th }}$ percentile thickness: 0.9 , without minor branches: 0.9). Moreover, the distribution was different: while the full model was skewed towards higher strain values, the model without minor branches was skewed towards lower values while both constant thickness models had a more symmetric distribution.

\section{Discussion}

In a recent work of our group, we set up a fluid-structure simulation framework on a mouse-specific geometry (14) with the ultimate goal to investigate the hypothesis that the interplay of hemodynamics and vascular wall mechanics plays a role in the initiation of dissecting AAs. The results showed that suprarenal side branches are a target location for stress concentrations, a finding that was in line with the location of medial tears that had been observed experimentally. But while this early work included some of the latest improvements in mouse-specific computational biomechanics such as (i) strongly coupled fluid-structure interaction, (ii) a high quality hexahedral mesh, (iii) a material model with parameters that were tuned to mouse-specific in-vivo measurements, and (iv) a backward displacement method to incorporate in-vivo circumferential stress, our FSI simulations were still subject to several limitations. We identified the following sources of uncertainty:

(i) The aortic geometry was based on a segmented 3D volume of the aortic lumen that was radially extended with a constant wall thickness (proportional to the local diameter). Current in-vivo imaging techniques (CT or MRI in humans, micro-CT in mice) do not yield sufficient resolution to include a detailed mouse-specific wall thickness into the model. Nevertheless a non-uniform thickness has been shown to have a significant influence on the outcome of numerical simulations $(22 ; 23 ; 24)$.

(ii) The aortic geometry did not include minor side branches such as subcostal arteries. As 
for the subject-specific thickness, the resolution of standard imaging approaches does not allow to visualize these minor branches. Nevertheless, it has been demonstrated that these small side branches are preferred regions for hemodynamic perturbations and vascular lesions development $(25 ; 26)$.

(iii) Axial pre-stretch was not incorporated into the model. This is a general limitation for models based on in-vivo images since, unlike circumferential prestresses, the axial stretch can only be calculated if one has access to the ex-vivo, zero-stretch condition of the vessel. Nevertheless, local variations in axial stretch and stress might have a significant influence in both the initiation and development of different cardiovascular diseases $(27 ; 28)$.

(iv) Simulations were only performed on a healthy, non-diseased aorta. While we did find stress concentrations near the same side branches where vascular damage had been observed in Ang II infused mice, a one-on-one check in which simulations and experiments were performed on the same animal is still lacking. Mice provide a unique opportunity to compare the computational hotspot predictions, since in a clinical setting there are typically no post-mortem scans available to investigate the predicted rupture risks.

(v) A simplified Arruda-Boyce material model was used rather than the more sophisticated 4-fiber family model (29) or Holzapfel material model (30). More complicated models generally need more parameters and are thus difficult to fit to in-vivo measurements. Moreover, while the anisotropic constitutive relations and fiber directions have been established ex-vivo (typically on idealized cylinder-shaped geometries), the in-vivo mechanical response at the bifurcation level is still unclear. Nevertheless, stress concentrations often occur near such bifurcations as they are the natural hotspots for disease initiation (13).

In this work we developed a semi-automatic morphing framework which represents, to the best of our knowledge, the first attempt to incorporate non-uniform axial stretch, non-uniform subjectspecific wall thickness and minor side branches within a computational model of a complex aortic structure. Combining the latest technology on in-vivo and ex-vivo small animal imaging, we advanced our modeling approach to include a level of detail that has not (yet) been reached by any model based on clinical images. Based on our results, we believe that the remarkable differences that were obtained in this manuscript provide important food for thought.

First of all, our results suggest that the constant thickness modelling assumption, which is standard in the field, may lead to incorrect results at particular bifurcations. Indeed, even if the overall strain field of both constant thickness models was similar to the outcome of the full model (Fig. 5), the hotspot of elevated strain near the celiac artery was significantly mitigated in both the lowest and the highest thickness model (Fig. 6). Since the error did not depend on the value of the thickness with respect to local diameter we hypothesize that the culprit in this specific case was the maximum inscribed diameter that was used for the local diameter calculation. Indeed, since the local wall thickness at each node is determined by the local diameter at that node, each bifurcation will have 
an abrupt transition zone between main branch (where thickness is higher) and side branch (where thickness is lower). A detailed comparison with the full model shows that at the level of the celiac artery this results in a wall thickness transition who significantly alters the strain distribution (Fig. $3 \mathrm{bc})$. This error is strongly dependent on the local branching pattern of the centerline on which the thickness algorithm is based, and would be difficult if not impossible to spot if no gold standard were available. This is in line with literature, where the use of constant thickness model has been shown to reduce the accuracy of AAA computational models $(22 ; 23 ; 24 ; 31)$.

A second important finding is that all side branches, including the very small ones, alter the local mechanical equilibrium of the aorta, both in axial and circumferential direction. Our results show that neglecting the contribution of the minor subcostal branches leads to a significant underestimation of the axial strain field in the dorsal region of the abdominal aorta, inducing an alteration of the strain hotspots in the celiac and mesenteric artery (Fig. 5, 6). This suggests that the tethered small vessels, which are attached to the surrounding structures such as the spine, provide a concrete mechanical support that must be considered when assessing in vivo axial stretch.

This implies that biomechanical simulations in which small (and sometimes even large) side branches are ignored probably make an important error when predicting the location of strain and/or stress hotspots. However, most in-vivo imaging techniques do not have sufficient resolution to include small branches into the models.

Closely related to the second finding, we conclude that the axial stretch in the murine aorta is highly non-uniform, and that this non-uniformity contributes to the distribution of stresses and strains along the aorta. These computational findings are in line with experimental results of Guo and Kassab, who reported a significant variation of the local axial stretch ratio along the thoracoabdominal aorta of $\mathrm{C57bl} / 6$ mice (32). The non-uniformity of the axial stretch has never been included in human models since (i) there are no experimental data reporting on the non-uniformity of the axial stretch in humans and (ii) most computational models do not have access to highresolution ex-vivo images of the aorta that would allow an account of axial stretch. But, similar to what is the case for small side branches, the fact that no data are available to prove it wrong does not necessarily imply that an assumption is correct. Interestingly, our observations are in line with the hypothesis that local axial variations of important mechanical properties, like stretch or stiffness, play an important role on both physiology and disease initiation on specific hotspots $(28 ; 33 ; 34)$.

Finally, we want to emphasize the limitations of our morphing approach. On the experimental side, biological tissue shrinkage due to sample fixation is a limitation well known in the literature $(35 ; 36)$. This may have affected the dimensions of the ex-vivo model, leading to a global overestimation of the strain field. Here, we assume that the fixation-related shrinkage occurs isotropically on the whole sample due to dehydratation. This is a limitation in our study, since a damage in the vascular wall may have a fundamentally different composition of materials (e.g. hydrophilic GAGs) that will retain different amounts of water. This is a limitation in our study, since a heterogeneous shrinking due to fixation might have affected the calculated hotspot distribution along the aorta. Another limitation is that the artificial extensions in the PCXTM mesh do not represent a real vessel and that 
the thickness in the distal region of the side branch may be overestimated. That is why the present framework is focused on the strain distribution in the main aorta, with particular focus on the celiac and mesenteric bifurcations.

On the modeling side, an Arruda-Boyce hyperelastic constitutive model has been used for the morphing simulation. While more sophisticated material models are available (37), their fitting to mouse data and subsequent implementation within the morphing framework have been shown problematic. In this respect it is important to keep in mind that for many cardiovascular applications it is much more important to correctly estimate the location of strain/stress hotspots than to get the absolute values right. Moreover, previous experimental studies highlighted that aortic wall stiffness shows an inverse correlation with wall thickness so that the global structural stiffness does not change along the aorta (38). This highlights the need for a mouse-specific heterogeneous constitutive model which, to our knowledge, has not been performed on any in vivo simulation of a complex vascular network. But as long as the material model is considered to be homogeneous, a change in that material model will only affect the values of the hotspots, not their locations. In this regard the heterogeneity of the constitutive model, particularly at the bifurcations, still represents a significant source of uncertainty and should be the object of further investigation.

In addition, the ex vivo PCXTM configuration provides a zero load but not a completely stress free configuration, since the circumferential pre-stretch can only be calculated by cutting and unloading the aortic wall. Future work should therefore focus on incorporating local stiffness variations and pre-stress into the model.

The strain concentrations that were found near celiac and mesenteric arteries are in agreement with the hypothesis that the medial tear that is formed at later stages of angiotensin II-infused mice originates from a mechanical imbalance at these 2 side branches (13). It would, however, be premature to interpret results as indicative for the initiation of dissecting aneurysms as we only considered a single case in this sensitivity analysis. In future work we aim to validate this novel computational framework in a large sample of mice, with the ultimate goal to evaluate the correlation of the predicted regions of maximal strain with contrast agent infiltration and imageguided histology.

In summary, this manuscript presents a novel methodological framework to evaluating the global strain field in the abdominal aorta while including unique features such as a detailed mouse-specific thickness, minor subcostal branches and non-uniform axial stretch. The key results of our study are the following: (i) constant thickness models based on the local diameter can yield unexpected spurious results near bifurcations; (ii) the support provided by the minor branches and the inclusion of non-uniform axial stretch significantly alters the computed strain field in the aorta; (iii) only a full model considering both mouse-specific thickness and minor branches can provide accurate results for the detection of hotspots potentially related to vascular damage. 


\section{BIBLIOGRAPHY}

1. Antiga, L., Steinman, D. A. Robust and objective decomposition and mapping of bifurcating vessels. IEEE transactions on medical imaging 23(6):704-713, 2004.

2. Antiga, L., Piccinelli, M., Botti, L., Ene-Iordache, B., Remuzzi, A., \& Steinman, D. A. An image-based modeling framework for patient-specific computational hemodynamics. Medical \& biological engineering \& computing 46(11):1097, 2008.

3. Avril, S., Badel, P., Gabr, M., Sutton, M. A., \& Lessner, S. M. Biomechanics of porcine renal arteries and role of axial stretch. Journal of biomechanical engineering 135(8):081007, 2013.

4. Bersi, M., Ferruzzi, J., Eberth, J., Gleason Jr, R., \& Humphrey, J. Consistent biomechanical phenotyping of common carotid arteries from seven genetic, pharmacological, and surgical mouse models. Annals of biomedical engineering 42(6):1207-1223, 2014.

5. Bols, J., Taelman, L., De Santis, G., Degroote, J., Verhegghe, B., Segers, P., \& Vierendeels, J. Unstructured hexahedral mesh generation of complex vascular trees using a multi-block grid-based approach. Computer methods in biomechanics and biomedical engineering 19(6):663-672, 2016.

6. Bond, A. R., Ni, C.-W., Jo, H., \& Weinberg, P. D. Intimal cushions and endothelial nuclear elongation around mouse aortic branches and their spatial correspondence with patterns of lipid deposition. American Journal of Physiology-Heart and Circulatory Physiology 298(2):H536--H544, 2010.

7. Collins, M., Bersi, M., Wilson, E., \& Humphrey, J. Mechanical properties of suprarenal and infrarenal abdominal aorta: implications for mouse models of aneurysms. Medical engineering \& physics 33(10):1262-1269, 2011.

8. Conlisk, N., Geers, A. J., McBride, O. M., Newby, D. E., \& Hoskins, P. R. Patient-specific modelling of abdominal aortic aneurysms: The influence of wall thickness on predicted clinical outcomes. Medical engineering \& physics 38(6):526-537, 2016.

9. Daugherty, A., \& Cassis, L. A. Mouse models of abdominal aortic aneurysms. Arteriosclerosis, thrombosis, and vascular biology 24(3):429-434, 2004.

10. Daugherty, A., Manning, M. W., \& Cassis, L. A. Angiotensin II promotes atherosclerotic lesions and aneurysms in apolipoprotein E--deficient mice. The Journal of clinical investigation 105(11):1605-1612, 2000.

11. Dua, M. M., \& Dalman, R. L. Hemodynamic influences on abdominal aortic aneurysm disease: Application of biomechanics to aneurysm pathophysiology. Vascular pharmacology 53(1):11-21, 2010.

12. Feintuch, A., Ruengsakulrach, P., Lin, A., Zhang, J., Zhou, Y.-Q., Bishop, J., . . others. Hemodynamics in the mouse aortic arch as assessed by MRI, ultrasound, and numerical modeling. American Journal of Physiology-Heart and Circulatory Physiology 292(2):H884--H892, 2007.

13. Figueroa, C. A., Baek, S., Taylor, C. A., \& Humphrey, J. D. A computational framework for fluid--solid-growth modeling in cardiovascular simulations. Computer methods in applied mechanics and engineering 198(45):35833602, 2009.

14. Friedman, J. H., Bentley, J. L., \& Finkel, R. A. An algorithm for finding best matches in logarithmic expected time. ACM Transactions on Mathematical Software (TOMS) 3(3):209-226, 1977.

15. Gamble, G., Beaumont, B., Smith, H., Zorn, J., Sanders, G., Merrilees, M., . . Sharpe, N. B-mode ultrasound images of the carotid artery wall: correlation of ultrasound with histological measurements. Atherosclerosis 102(2):163$173,1993$. 
16. Gasser, T. C., Auer, M., Labruto, F., Swedenborg, J., \& Roy, J. Biomechanical rupture risk assessment of abdominal aortic aneurysms: model complexity versus predictability of finite element simulations. European Journal of Vascular and Endovascular Surgery 40(2):176-185, 2010.

17. Guo, X., \& Kassab, G. S. Variation of mechanical properties along the length of the aorta in C57bl/6 mice. American Journal of Physiology-Heart and Circulatory Physiology 285(6):H2614-H2622, 2003.

18. Haker, S., Angenent, S., Tannenbaurn, A., Kikinis, R. Nondistorting flattening maps and the 3-D visualization of colon CT images. IEEE Transactions on Medical Imaging 19(7):665-670, 2000.

19. Holzapfel, G. A., Gasser, T. C., Ogden, R. W. A new constitutive framework for arterial wall mechanics and a comparative study of material models. Cardiovascular soft tissue mechanics 1-48, 2001.

20. Humphrey, J. D., \& Holzapfel, G. A. Mechanics, mechanobiology, and modeling of human abdominal aorta and aneurysms. Journal of biomechanics 45(5):805-814, 2012.

21. Humphrey, J., Eberth, J., Dye, W., Gleason, R. Fundamental role of axial stress in compensatory adaptations by arteries. Journal of biomechanics 42(1):1-8, 2009.

22. Kim, J., Baek, S. Circumferential variations of mechanical behavior of the porcine thoracic aorta during the inflation test. Journal of biomechanics 44(10):1941-1947, 2011.

23. Nakashima, Y., Plump, A. S., Raines, E. W., Breslow, J. L., Ross, R. (1994). ApoE-deficient mice develop lesions of all phases of atherosclerosis throughout the arterial tree. Arteriosclerosis, thrombosis, and vascular biology 14(1):133-140, 1994.

24. Raaz, U., Zöllner, A. M., Schellinger, I. N., Toh, R., Nakagami, F., Brandt, M., . . Adam, M. a. Segmental aortic stiffening contributes to experimental abdominal aortic aneurysm development. Circulation, 2015.

25. Raut, S. S., Jana, A., De Oliveira, V., Muluk, S. C., \& Finol, E. A. The importance of patient-specific regionally varying wall thickness in abdominal aortic aneurysm biomechanics. Journal of biomechanical engineering 135(8):081010, 2013.

26. Reymond, P., Crosetto, P., Deparis, S., Quarteroni, A., \& Stergiopulos, N. Physiological simulation of blood flow in the aorta: comparison of hemodynamic indices as predicted by 3-D FSI, 3-D rigid wall and 1-D models. Medical engineering \& physics 35(6):784-791, 2013.

27. Saraff, K., Babamusta, F., Cassis, L. A., \& Daugherty, A. Aortic dissection precedes formation of aneurysms and atherosclerosis in angiotensin II-infused, apolipoprotein E-deficient mice. Arteriosclerosis, thrombosis, and vascular biology 23(9):1621-1626, 2003.

28. Shang, E. K., Nathan, D. P., Woo, E. Y., Fairman, R. M., Wang, G. J., Gorman, R. C., . . Jackson, B. M. Local wall thickness in finite element models improves prediction of abdominal aortic aneurysm growth. Journal of vascular surgery 61(1):217-223, 2015.

29. Stampanoni, M., Borchert, G., Wyss, P., Abela, R., Patterson, B., Hunt, S., . . Ruegsegger, P. High resolution X-ray detector for synchrotron-based microtomography. Nuclear Instruments and Methods in Physics Research Section A: Accelerators, Spectrometers, Detectors and Associated Equipment 491(1): 291-301, 2002.

30. Trachet, B., Bols, J., Degroote, J., Verhegghe, B., Stergiopulos, N., Vierendeels, J., \& Segers, P. An animal-specific FSI model of the abdominal aorta in anesthetized mice. Annals of biomedical engineering 43(6):1298-1309, 2015.

31. Trachet, B., Fraga-Silva, R. A., Piersigilli, A., Tedgui, A., Sordet-Dessimoz, J., Astolfo, A., . . Stergiopulos, N. 
Dissecting abdominal aortic aneurysm in Ang II-infused mice: suprarenal branch ruptures and apparent luminal dilatation. Cardiovascular research 105(2):213-222, 2015.

32. Trachet, B., Piersigilli, A., Aslanidou, L., Fraga-Silva, R. A., Sordet-Dessimoz, J., Villanueva-Perez, P., . . Segers, P. Angiotensin II infusion into ApoE-/- mice: a model for aortic dissection rather than abdominal aortic aneurysm? Cardiovascular Research 113(10):1230-1242, 2017.

33. Trachet, B., Renard, M., De Santis, G., Staelens, S., De Backer, J., Antiga, L., . . Segers, P. An integrated framework to quantitatively link mouse-specific hemodynamics to aneurysm formation in angiotensin II-infused ApoE-/mice. Annals of biomedical engineering 39(9):2430-2444, 2011.

34. Vincent, P., Plata, A., Hunt, A., Weinberg, P., \& Sherwin, S. Blood flow in the rabbit aortic arch and descending thoracic aorta. Journal of The Royal Society Interface 8(65):1708-1719, 2011.

35. Vorp, D. A. Biomechanics of abdominal aortic aneurysm. Journal of biomechanics, 40(9):1887-1902, 2007.

36. Voß, S., Glaßer, S., Hoffmann, T., Beuing, O., Weigand, S., Jachau, K., . . Berg, P. Fluid-Structure Simulations of a Ruptured Intracranial Aneurysm: Constant versus Patient-Specific Wall Thickness. Computational and Mathematical Methods in Medicine, 2016.

37. Wehrl, H. F., Bezrukov, I., Wiehr, S., Lehnhoff, M., Fuchs, K., Mannheim, J. G., . . Pichler, B. J. Assessment of murine brain tissue shrinkage caused by different histological fixatives using magnetic resonance and computed tomography imaging. Histol Histopathol 30(5):601-13, 2015.

38. Wilson, J. S., Bersi, M. R., Li, G., \& Humphrey, J. D. Correlation of Wall Microstructure and Heterogeneous Distributions of Strain in Evolving Murine Abdominal Aortic Aneurysms. Cardiovascular Engineering and Technology 8(2):1-12, 2017.

39. Wilson, J., Baek, S., \& Humphrey, J. Importance of initial aortic properties on the evolving regional anisotropy, stiffness and wall thickness of human abdominal aortic aneurysms. Journal of The Royal Society Interface, 2012.

\section{Tables}

\begin{tabular}{|l|c|c|c|}
\hline \multicolumn{1}{|c|}{ Mesh } & Number of nodes & Number of elements & Number of elements through the thickness \\
\hline Full model & 916660 & 730832 & 4 \\
\hline Constant thickness (30\%) & 916660 & 730832 & 4 \\
\hline Constant thickness (47\%) & 916660 & 730832 & 4 \\
\hline No minor branches & 672715 & 536724 & 4 \\
\hline
\end{tabular}




\section{Figures}

EX-VIVO PCXTM

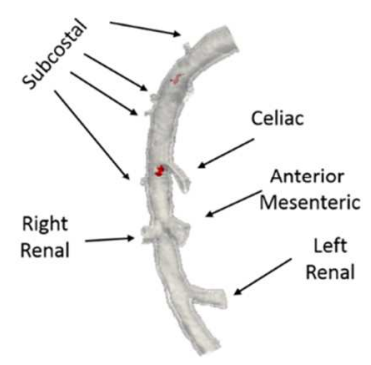

LENGTH SCALE $1 \mathrm{~mm}$

(a)
IN-VIVO CT

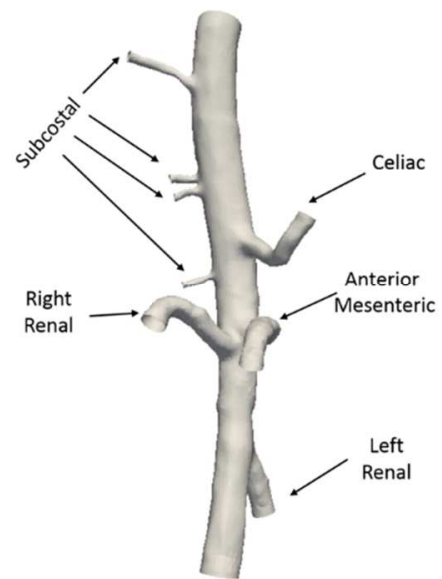

(b)
BRANCH EXTENSION

HEXAHEDRAL MESH

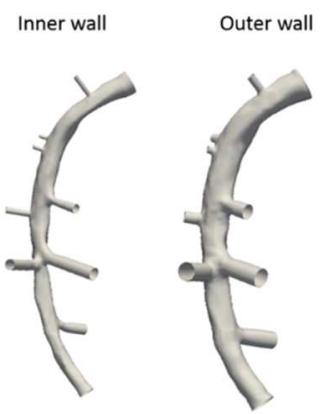

(c)

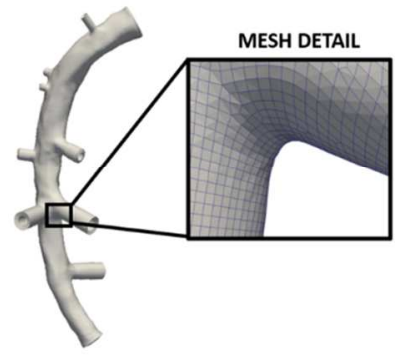

(d)

FIGURE 1. PREPROCESSING PHASE: (A) PCXTM MODEL (CONTRAST AGENT INFILTRATIONS ARE HIGHLIGTED IN RED); (B) CT MODEL; (C) INNER AND OUTER WALLS OF THE PCXTM AFTER ARTIFICIAL BRANCH EXTENSION; (D) CONFORMAL HEXAHEDRAL MESH WITH MOUSE SPECIFIC THICKNESS. BRANCH SPLITTING CIRCUMFERENTIAL

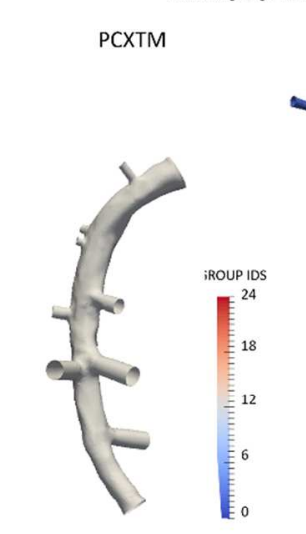

LENGTH SCALE $1 \mathrm{~mm}$

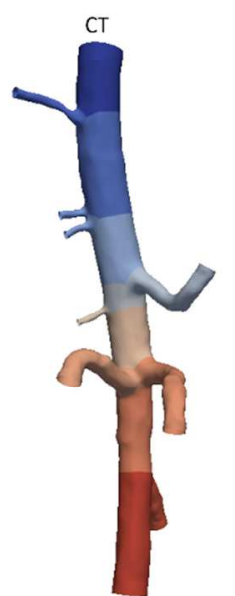
LONGITUDINAL

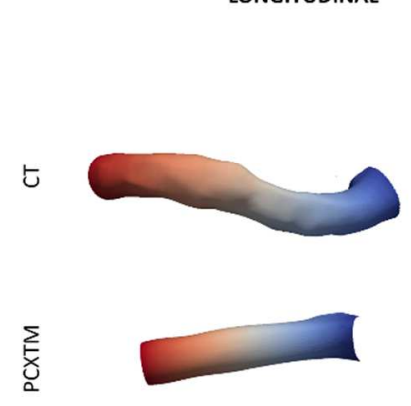

(c)

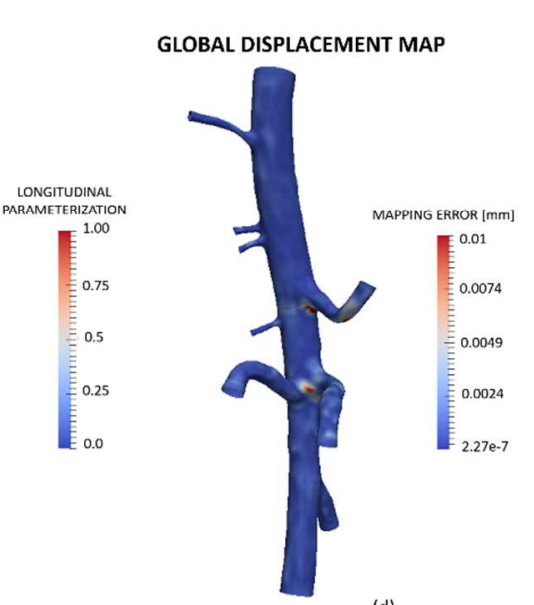

(d)

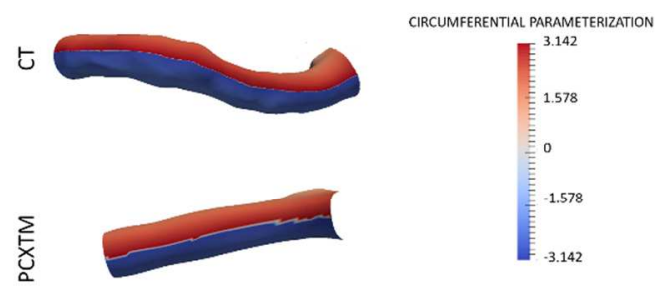

(b)

MORPHING FE SIMULATION

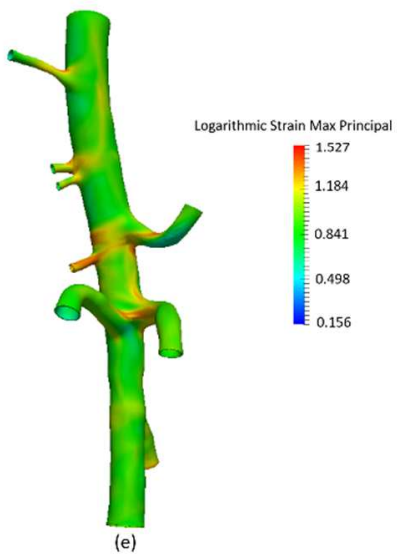

FIGURE 2. MORPHING FRAMEWORK SET-UP: (A) BRANCH SPLITTING; (B) CIRCUMFERENTIAL MAPPING COMPUTATION; (C) LONGITUDINAL MAPPING COMPUTATION; (D) GLOBAL DISPLACEMENT MAP ERROR CONTOUR PLOT WITH RESPECT 
TO THE ORIGINAL CT MODEL; (E) MAX PRINCIPAL STRAIN CONTOUR PLOT AFTER MORPHING FE SIMULATION.

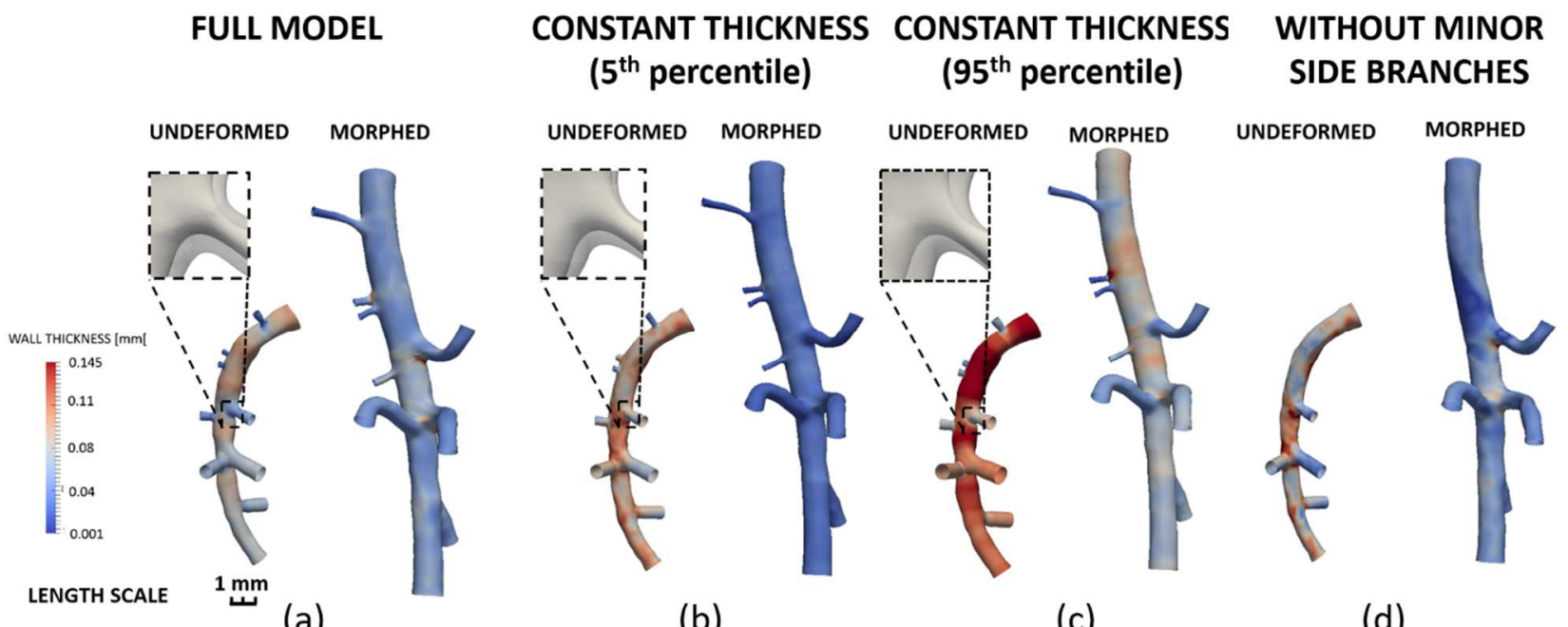

FIGURE 3. WALL THICKNESS DISTRIBUTION BEFORE AND AFTER MORPHING SIMULATION: (A) FULL MODEL; (B) $5^{\text {TH }}$ PERCENTILE CONSTANT THICKNESS, (C) $95^{\mathrm{TH}}$ PERCENTILE CONSTANT THICKNESS (D) WITHOUT MINOR SIDE BRANCHES.

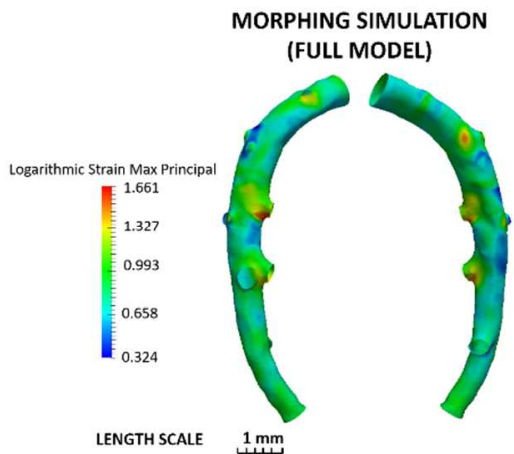

(a)

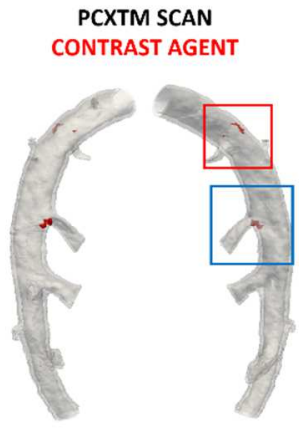

(b)

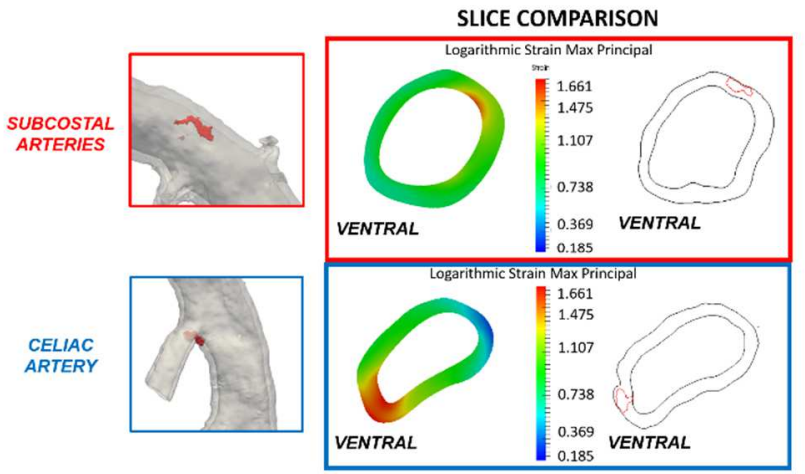

(c)

FIGURE 4. PCXTM QUALITATIVE COMPARISON: (A) LOGARITHMIC STRAIN MAX PRINCIPAL CONTOUR PLOT USING THE FULL MODEL; (B) ORIGINAL PCXTM MODEL (CONTRAST AGENT INFILTRATION ARE HIGHLIGHTED IN RED); (C) 2D SLICES COMPARISON WITH FOCUS ON THE REGIONS OF CONTRAST AGENT INFILTRATIONS. 

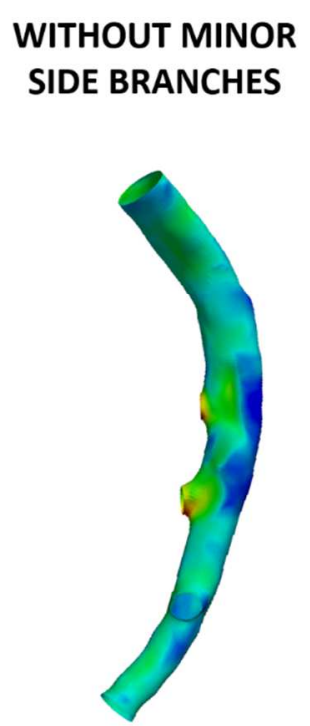

\section{LENGTH SCALE $\quad 1 \mathrm{~mm}$}

(a)

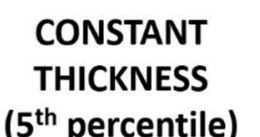

(5 $5^{\text {th }}$ percentile)

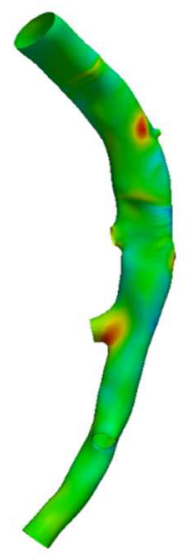

(b)

\section{CONSTANT \\ THICKNESS}

(95 ${ }^{\text {th }}$ percentile)

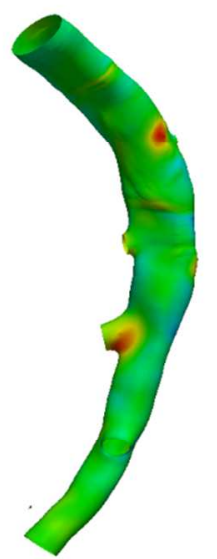

FULL

MODEL

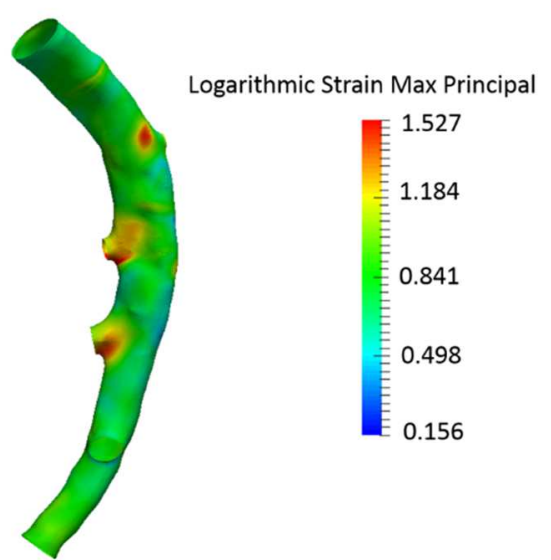

FIGURE 5. SENSITIVITY ANALYSIS: LOGARITHMIC STRAIN MAX PRINCIPAL CONTOUR PLOT USING, (A) MODEL WITHOUT MINOR SIDE BRANCHES, (B) $5^{\mathrm{TH}}$ PERCENTILE CONSTANT THICKNESS, (C) $95^{\mathrm{TH}}$ PERCENTILE CONSTANT THICKNESS, (D) FULL MODEL.
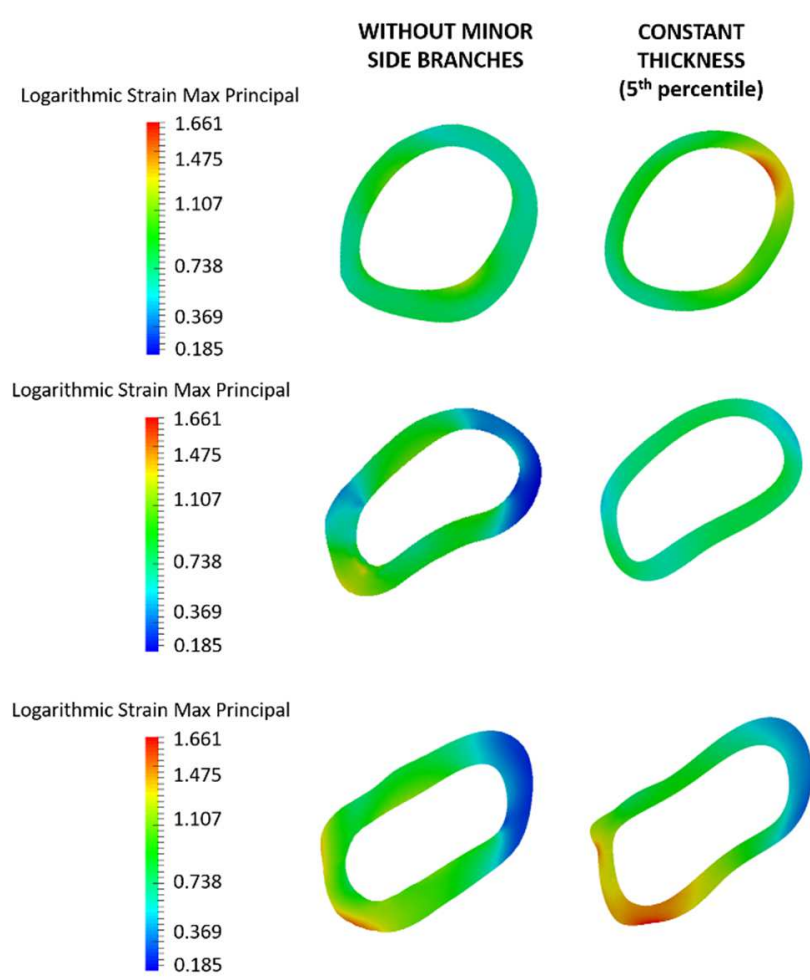
CONSTANT
(95 ${ }^{\text {th }}$ percentile)
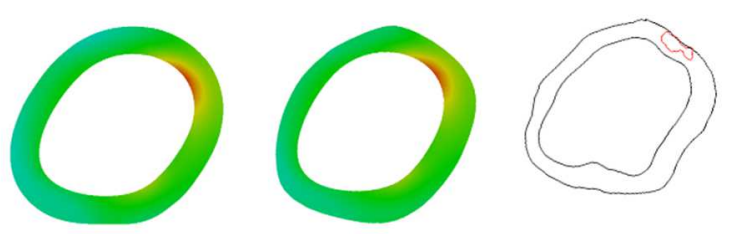

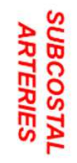
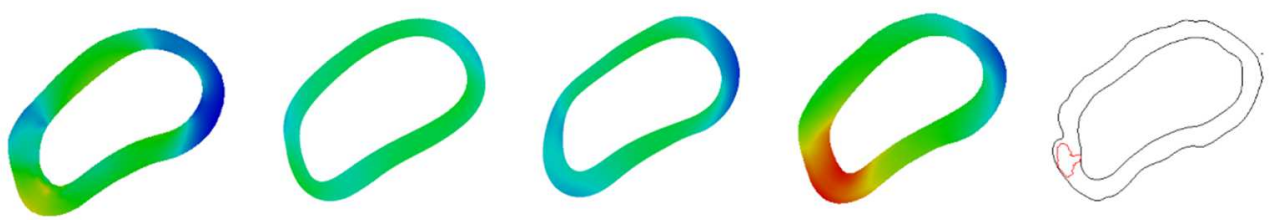

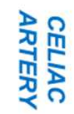
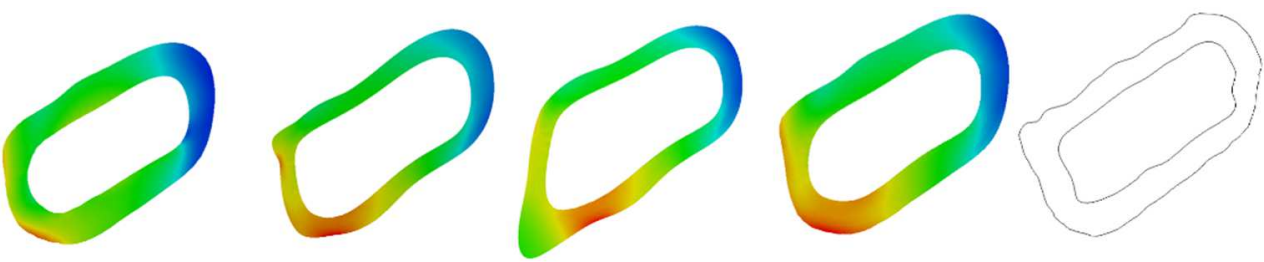

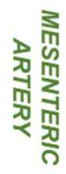

(a)

(b)

(c)

(d)

(e)

FIGURE 6 2D SLICE STRAIN MAX PRINCIPAL CONTOUR PLOT AT THE STRAIN HOTSPOTS LOCATIONS, (A) MODEL WITHOUT MINOR SIDE BRANCHES, (B) $5^{\mathrm{TH}}$ PERCENTILE CONSTANT THICKNESS, (C) $95^{\mathrm{TH}}$ PERCENTILE CONSTANT THICKNESS, (D) FULL MODEL, (E) PCXTM SCAN (CONTRAST AGENT HIGHLIGHTED IN RED). 


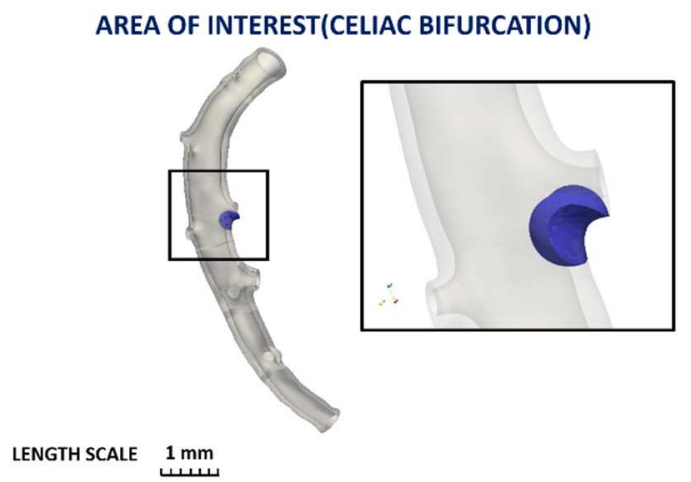

(a)

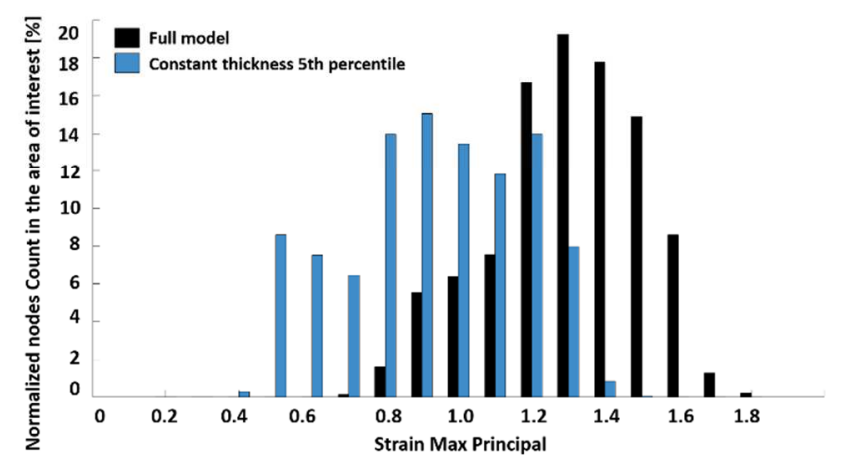

(c)

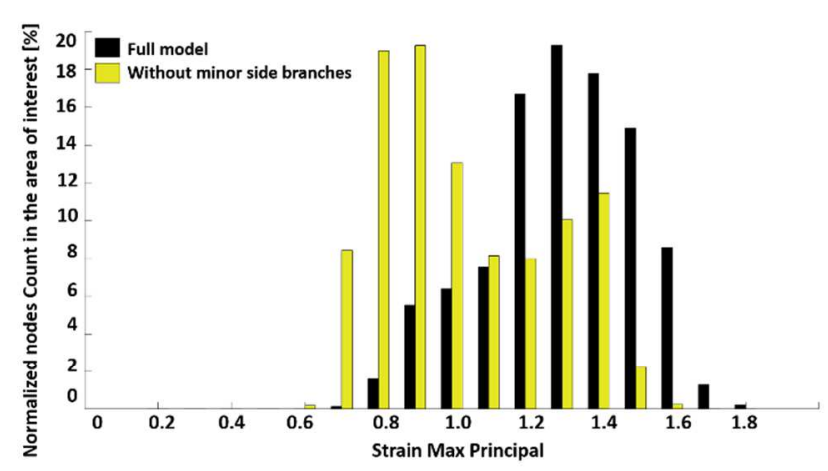

(b)

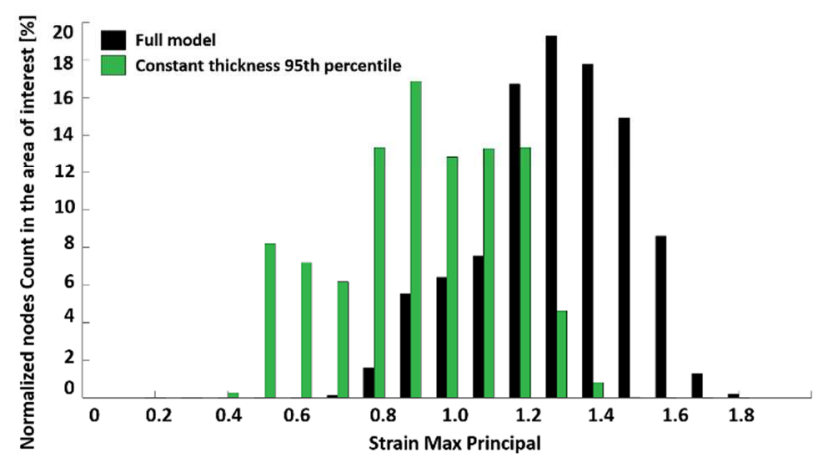

(d)

FIGURE 7 QUANTITATIVE COMPARISON: (A) AREA OF INTEREST USED FOR THE EVALUATION, (B-C-D) HISTOGRAMS OF THE STRAIN MAX PRINCIPAL DISTRIBTION USING THE MODEL WITHOUT SIDE BRANCHES (B), ${ }^{\mathrm{TH}}$ PERCENTILE CONSTANT THICKNESS (C), 95 ${ }^{\mathrm{TH}}$ PERCENTILE CONSTANT THICKNESS. STRAIN DISTRIBUTION OF THE FULL MODEL HAS BEEN OVERLAPPED IN ALL THE HISTOGRAMS. 